\title{
Fuzzy-Enforced Complementarity Constraints in Nonlinear Interior Point Method-Based Optimization
}

\section{Hwachang Song}

Department of Electrical and Information Engineering, Seoul National University of Science \& Technology, Seoul, Korea

\section{]jfis}

\begin{abstract}
This paper presents a fuzzy set method to enforce complementarity constraints (CCs) in a nonlinear interior point method (NIPM)-based optimization. NIPM is a Newton-type approach to nonlinear programming problems, but it adopts log-barrier functions to deal with the obstacle of managing inequality constraints. The fuzzy-enforcement method has been implemented for CCs, which can be incorporated in optimization problems for real-world applications. In this paper, numerical simulations that apply this method to power system optimal power flow problems are included.
\end{abstract}

Keywords: Complementarity constraints, Fuzzy enforcement, Nonlinear interior point methods, Nonlinear optimization, Optimal power flow

\section{Introduction}

In real-world application, several practical situations can be modeled using nonlinear optimization problems with system equilibrium constraints, including complementarity conditions [1-3]. In optimization problems, complementarity constraints (CCs) are used to explain the disjunctive condition of system models [4]; that is, depending on a given environment, different rules are imposed for systems with these constraints. They can be found in mechanics, circuits with electronic switching devices, structural studies, and power market models in the electricity industry $[1,2,5]$.

In the past decades, many research activities on the application of interior point methods

Received: May. 31, 2013

Revised : Sep. 10, 2013

Accepted: Sep. 13, 2013

Correspondence to: Hwachang Song (hcsong@seoultech.ac.kr)

(CThe Korean Institute of Intelligent Systems

(c) This is an Open Access article distributed under the terms of the Creative Commons Attribution Non-Commercial License (http://creativecommons.org/licenses/ by-nc/3.0// which permits unrestricted noncommercial use, distribution, and reproduction in any medium, provided the original work is properly cited. (IPMs) to nonlinear programming, one of the diverse engineering problems, have been conducted [6-10]. IPM adopts the log-barrier penalty functions to adequately cope with inequality constraints; it was first proposed by Frish [11]. The IPM presented by Karmarkar [12] was designed for linear programming problems, and it was considered to be approximately 50 times faster than the common simplex method. Later, IPMs were devised [6, 7, 13, 14], which can find solutions to primal and dual variables. The current paper mainly discusses the methods for dealing with complementarity conditions in primal and dual nonlinear IPMs (NIPMs).

The standard complementarity problems find solutions to the following conditions:

$$
x \geq 0, F(x) \geq 0,\langle F(x), x\rangle=0
$$

where the operator of the last condition in (1) denotes the inner products. In [15], a method of 
formulating generalized complementarity problems with an unconstrained optimization problem was discussed. From the result, an optimization problem with complementarity conditions was envisaged to be reformulated into a multi-objective optimization problem. For practical NIPM applications, a set of reduced correction equations is usually adopted, including the Hessian term and the Jacobian sub-matrix, only for the equality constraints $[16,17]$. Thus, if the equality formulation of (1) is added to the NIPM formulation, the system size of the correction equations must be increased; hence, a certain amount of effort is required for modification of the $\mathrm{CC}$.

The present paper presents a method of fuzzy-enforcing CC as a form of inequality for NIPM-based optimization methods. Fuzzy enforcement was originally proposed in [18], but it was designed for general equality and inequality constraints in successive linear programming algorithms. By introducing fuzzy enforcement, the method proposed in this paper can adequately deal with the concept of "not too much" violation of the complementarity conditions, providing enough room for the solutions to approach optimality. This paper also presents the numerical results when the method was applied to an NIPM-based optimal power flow (OPF) with CCs in the generator voltage and reactive power generation.

\section{Fuzzy Enforcement of CCs}

\subsection{Problem Formulation}

The formulation of nonlinear programming problems in this study with the $\mathrm{CC}$ of interest can be briefly expressed as follows:

$$
\begin{array}{ll}
\min & f(x) \\
\text { s.t. } & g(x)=0 \\
& h_{\min } \leq h(x) \leq h_{\max } \\
& \left(c_{i}\left(x_{i}\right)-\alpha_{i}\right)\left(x_{i}-\beta_{i}\right)=0 \\
& c_{i}\left(x_{i}\right)-\alpha_{i} \geq 0, x_{i}-\beta_{i} \geq 0, \\
& i=1, \ldots, k c
\end{array}
$$

where $x$ is the vector that includes the control and dependent variables. In (2), $f($ ?) is the objective function; $g($ ?) and $h($ ?) are the function vectors for the equality and inequality constraints, respectively; and $h_{\min }$ and $h_{\max }$ denote the lower and upper limits of $h\left(\right.$ ?), respectively. $x_{i}$ stands for the $i$ th variable of $x$ involved in the CC; $c\left(x_{i}\right)-\alpha_{i}$ and $x_{i}-\beta_{i}$ are the functions for the complementarity conditions, and they are non-negative; and $k c$ is the number of CCs in the problem.

On the basis of the condition where the $\mathrm{CC}$ factors are non-

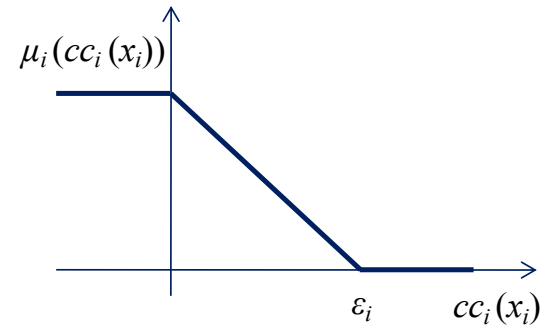

Figure 1. Membership function for the inequality form of a CC.

negative, the equivalent inequality constraint can be expressed as follows:

$$
\left(c_{i}\left(x_{i}\right)-\alpha_{i}\right)\left(x_{i}-\beta_{i}\right) \leq 0
$$

When NIPM is applied to the optimization problem using this inequality form of the $\mathrm{CC}$, using the same dimensional correction equations as those of the nonlinear optimization problem is possible without the equality form of the CC. However, NIPM employs the log-barrier functions to force the solution in the whole procedure within the feasible region; hence, the solution cannot move from the initial vector of $x$ to find better solutions in terms of optimality and feasibility. Thus, a facilitating technique to provide enough room for moving the solutions might be needed, which takes into consideration the CC condition.

\subsection{Fuzzy Enforcement of the CCs}

We let the $i$ th CC function in (3) be $c c_{i}\left(x_{i}\right)$. The fuzzy set theory [19] can be applied to the CC because during the NIPM solution process, the "not too much" violation of a CC might be acceptable. From the fuzzy relation, the inequality form of the $\mathrm{CC}$ can be written as

$$
c c_{i}\left(x_{i}\right) \tilde{\leq} 0
$$

Each fuzzy relation in the fuzzy set theory is associated with a membership function that represents the degree of certainty. The membership function in (4) can be expressed as follows (Figure 1):

$$
\mu_{i}\left(c c\left(x_{i}\right)\right)=\left\{\begin{array}{cc}
1, & c c_{i}\left(x_{i}\right) \leq 0 \\
\left(\varepsilon_{i}-c c_{i}\left(x_{i}\right)\right) / \varepsilon_{i}, & 0 \leq c c_{i}\left(x_{i}\right) \leq \varepsilon_{i} \\
0, & c c_{i}\left(x_{i}\right)>\varepsilon_{i}
\end{array}\right.
$$

where $\varepsilon_{i}$ stands for the acceptable limit of violating the $i$ th CC during the solution process.

To apply the fuzzy-enforced CC to the optimization problem, the degree of satisfaction in the NIPM solution process should 
be enhanced. For this purpose, the optimization problem can be rewritten with a multi-objective function as follows:

$$
\begin{array}{ll}
\min & f(x)-a_{c c} \sum_{i} z_{i} \\
\text { s.t. } & g(x)=0 \\
& \underline{h}_{\min } \leq \underline{h}(x) \leq \underline{h}_{\max } \\
& z_{i} \leq \mu_{i}\left(c c_{i}\left(x_{i}\right)\right) \\
& i=1, \ldots, k c
\end{array}
$$

where $a_{c c}$ is the weighting factor for the term that maximizes $z_{i}$, which is the lower limit of the membership function of the $i$ th CC. In $[6, \underline{h}(\cdot)$ is the function vector for the inequality constraints, including the non-negativity condition of the CC factors, and $\underline{h}_{\min }$ and $\underline{h}_{\max }$ denote the lower and upper limit vectors of $\underline{h}(\cdot)$, respectively.

The second term of the objective function in (6) forces each $z_{i}$ to the reachable maximum value of its membership function, and $z_{i}$ is the lower limit of the membership function. From (6), we can notice that the selection of $a_{c c}$ is quite important. Similar to the membership function, the maximum value of $z_{i}$ is one. If the slope of the original objective function $f(x)$ is much higher than $a_{c c}$, then $z_{i}$ approaches one, and hence, the infeasibility of the CCs might not be acceptable. Thus, keeping $a_{c c}$ around the maximum value of $f(x)$ during the solution process would be better.

\subsection{Application of NIPM}

To solve the nonlinear optimization problem in (6), this study adopts the NIPM $[7,17]$ using the fuzzy-enforced CCs. NIPM is a Newton-type approach to nonlinear programming problems, but the introduction of log-barrier functions for the inequality constraints facilitates the dealing of these constraints. In addition, a step size of each solution process is selected to force the solution within the feasible region; thus, deciding the set of active inequality constraints, which is usually needed in Newton-type approaches, becomes unnecessary.

As mentioned in subsection 2.1, the two CC factors are nonnegative. Therefore, the lower limit of $c c_{i}\left(x_{i}\right)$ is zero. In addition, we assume that the maximum $c c_{i}\left(x_{i}\right)$ is $\varepsilon_{i}$. On the basis of this assumption, only the middle section of the membership function is needed, and the inequality function of the $i$ th CC can be expressed as follows:

$$
0 \leq c c_{i}\left(x_{i}\right)+\varepsilon_{\mathrm{i}} z_{i} \leq \varepsilon_{\mathrm{i}}
$$

When $z_{i}$ is zero, $c c_{i}\left(x_{i}\right)$ varies in the range $\left[0, \varepsilon_{i}\right]$; when $z_{i}$ is one, $c c_{i}\left(x_{i}\right)$ should be zero. When the NIPM is applied, the solution in the middle of the process should be within the feasible region; thus, using (7) is adequate for the $\mathrm{CC}$ inequality constraints.

For the NIPM application, the problem formulation can be modified as follows:

$$
\begin{array}{ll}
\min & f(x)-a_{c c} Z e \\
\text { s.t. } & g(x)=0 \\
& \underline{h}(x)-\mathrm{s}_{\mathrm{L}}-\underline{h}_{\min }=0 \\
& \underline{h}(x)+\mathrm{s}_{\mathrm{U}}-\underline{h}_{\max }=0 \\
& c c(x)+Z E e-s_{C L}=0 \\
& c c(x)+Z E e+s_{C U}-E e=0 \\
& s_{L}, s_{U}, s_{C L}, s_{C U} \geq 0
\end{array}
$$

where $Z$ and $E$ are diagonal matrices whose $(i, i)$ components are $z_{i}$ and $\varepsilon_{i}$, respectively. In $(8), s_{L}$ and $s_{U}$ are the vectors with slack variables for the upper and lower constraints of $\underline{h}($ ?), respectively; $s_{C L}$ and $s_{C L}$ are the vectors with slack variables for the upper and lower constraints of the CCs, respectively; $c c(x)$ is the function vector, including the entire CCs; and $e$ is a vector whose component values are all one. The slack variables convert the inequality constraints into equality constraints.

When the log-barrier functions are considered for the remaining inequality constraints of the slack variables, the Lagrange function can be constructed as follows:

$$
\begin{aligned}
& \mathrm{L}\left(x, z, \mathrm{~s}_{\mathrm{L}}, \mathrm{s}_{\mathrm{U}}, s_{C L}, s_{C U} ; \lambda, \pi_{L}, \pi_{U}, \pi_{C L}, \pi_{C U}\right) \\
& =f(x)-a_{c c} Z e-\lambda^{T} g(x) \\
& -\pi_{L}^{T}\left(\underline{h}(x)-\mathrm{s}_{\mathrm{L}}-\underline{h}_{\min }\right) \\
& -\pi_{U}^{T}\left(\underline{h}(x)+\mathrm{s}_{\mathrm{U}}-\underline{h}_{\max }\right) \\
& \quad-\pi_{C L}^{T}\left(c c(x)+Z E e-s_{C L}\right) \\
& \quad-\pi_{C U}^{T}\left(c c(x)+Z E e+s_{C U}-E e\right) \\
& \quad-\mu p\left(\mathrm{~s}_{\mathrm{L}}, \mathrm{s}_{\mathrm{U}}, s_{C L}, s_{C U}\right) \\
& p\left(\mathrm{~s}_{\mathrm{L}}, \mathrm{s}_{\mathrm{U}}, s_{C L}, s_{C U}\right) \\
& \equiv \sum_{i} \ln \left(s_{L i}\right)+\sum_{i} \ln \left(s_{U i}\right) \\
& \quad+\sum_{k} \ln \left(s_{C L k}\right)+\sum_{k} \ln \left(s_{C U k}\right)
\end{aligned}
$$

where $\lambda$ is the Lagrangian multipliers for the equality constraints and $g()=$.0 . In (6), $\pi_{L}$ and $\pi_{U}$ are the Lagrangian multipliers of the lower and upper limits of $\underline{h}\left(\right.$.), respectively; $\pi_{C L}$ and $\pi_{C U}$ are the lower and upper limits of the fuzzy-enforced CCs, respectively; $p($.) is the summation of the log-barrier functions, which keeps the solution within the feasible region; and 
$\mu$ is a barrier parameter.

\subsection{Implementation of fuzzy enforcement of CCs on OPFs}

In this study, the fuzzy enforcement of a CC was applied to the problem of generator voltage and reactive power generation in the formulation of the OPF. The CC in the OPF formulation can be expressed as follows:

$$
\begin{aligned}
& \left(Q_{i, \text { max }}-Q_{i}\right)\left(V_{i, \text { ref }}-V_{i}\right)=0 \\
& \left(Q_{i}-Q_{i, \text { min }}\right)\left(V_{i}-V_{i, r e f}\right)=0
\end{aligned}
$$

where $Q_{i, \max }$ and $Q_{i, \min }$ denote the upper and lower limits of the reactive power generation at bus $i, Q_{i}$, respectively. In (10) and (11), $V_{i}$ and $V_{i, r e f}$ are the generator terminal voltage and its reference value at bus $i$, respectively.

The OPF formulation must satisfy only (10) or [11), that is, when the CC in (10) is taken into account, that of (11) should not be included in the formulation. Even though the above CC must be considered, the conventional OPF does not include the $\mathrm{CC}$ for the generator voltage and the reactive power generation. In this study, fuzzy enforcement is adopted for the CC in (10) and (11), and the NIPM process decides which CC is to be incorporated into the formulation.

\section{Numerical Examples}

This section presents an illustrative example using an island power system. The system includes nine in-service generating units, three wind turbine generators, battery energy storage systems (BESSs), and two high voltage direct current (HVDC) interties from external electric network systems to support the electricity consumption level of the system. In the simulation, we assume that the active and reactive power injections from the HVDC interties are fixed. Further, the wind turbine generators are considered as conventional generators with reactive power capability. The voltage stability issues of the Jeju system were described in [20].

In the present study, the fuzzy-enforced CCs for the generator voltages and reactive power generation are implemented on a modified version of the NIPM-based OPF in [17]. In the simulation, the objective function of the OPF is to minimize the total loss in the transmission lines. In the commonly used OPFs, the control vectors are the desired voltage magnitudes of the generators. However, the target of the OPF proposed in this paper is to determine the reactive power output of the

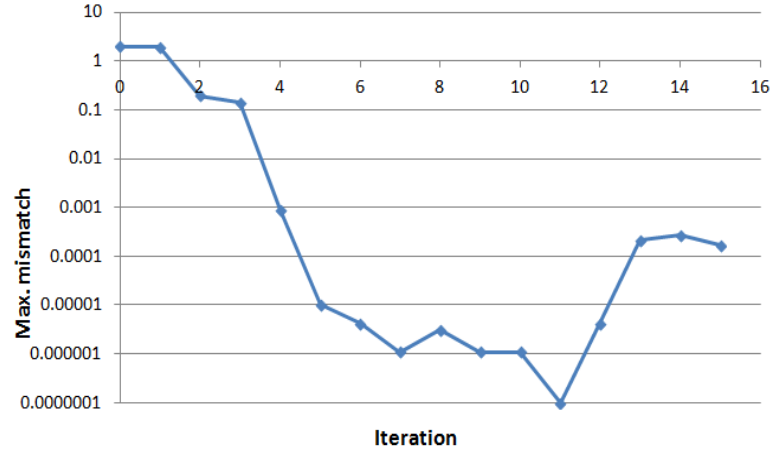

Figure 2. Change in complementary gap during the solution process.

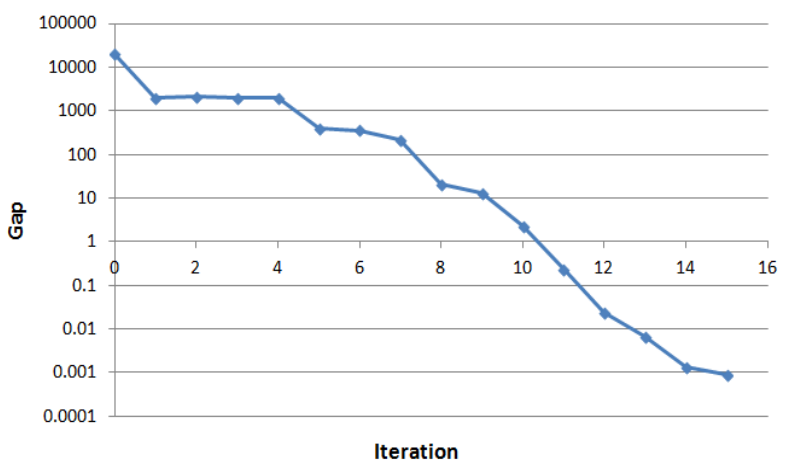

Figure 3. Change in maximum mismatch during the solution process.

BESS without any changes in the generator terminal voltages. The current control settings of the generator voltages can be maintained by considering their CCs, as expressed in 97 and (10). To reduce the transmission loss, only the reactive power output of the BESS in the power system is allowed to move as control vectors.

For the island power system, the NIPM-based OPF with the fuzzy-enforced CC for the generators was performed. The process took 16 iterations until the solution satisfied the stopping criteria. The maximum allowable mismatch for the equality constraints was $10^{2}$, and the tolerance of the complementary gap for the primal and dual variables in the simulation was $10^{-3}$. Figures 2 and 3 show the change in the complementary gap and the maximum mismatch of the network constraints, respectively.

Figure 2 shows that the complementary gap is gradually reduced until it becomes less than the tolerance. The centering parameter [16] of the NIPM is set to 0.1 , and hence, we can expect the complementary gap to reduce by $90 \%$. Figure 3 shows that in the initial part of the solution process, the maximum mismatch is dramatically reduced below the solution tolerance, but in further iteration, the maximum mismatch increases to 
Table 1. QV output of the generators at the final solution

\begin{tabular}{lcccccc}
\hline Bus\# & $\mathrm{Q}[\mathrm{pu}]$ & $\mathrm{V}[\mathrm{pu}]$ & $\mathrm{V}_{\text {ref }}[\mathrm{pu}]$ & $\mathrm{Q}_{\max }[\mathrm{pu}]$ & $\mathrm{Q}_{\min }[\mathrm{pu}]$ & $\mathrm{CC}$ error \\
\hline 5 & 0.048584 & 1.024289 & 1.025 & 0.05 & -0.25 & $1.00666 \mathrm{E}-06$ \\
55 & 0.029888 & 1.030468 & 1.025 & 0.04 & -0.24 & 0.001475749 \\
555 & -0.01021 & 1.025782 & 1.025 & 0.0075 & -0.025 & $1.15683 \mathrm{E}-05$ \\
20121 & -0.05866 & 1.006287 & 1 & 0.35 & -0.1 & 0.000259928 \\
20122 & -0.07507 & 1.006723 & 1 & 0.35 & -0.1 & 0.000167573 \\
20124 & 0.120517 & 1.007318 & 1 & 0.363 & -0.25 & 0.002711446 \\
20126 & -0.03289 & 1.004337 & 1 & 0.048 & -0.1 & 0.000291063 \\
20127 & -0.03192 & 1.004232 & 1 & 0.048 & -0.1 & 0.000288117 \\
20173 & -0.02802 & 1.002534 & 1 & 0.5 & -0.12 & 0.00023308 \\
20174 & 0.097852 & 1.005073 & 1 & 0.363 & -0.12 & 0.001105161 \\
22140 & -0.05482 & 1.005461 & 1 & 0.5 & -0.1 & 0.000246719 \\
22190 & -0.02301 & 1.004091 & 1 & 0.5 & -0.1 & 0.000314985 \\
\hline
\end{tabular}

some extent and then decreases below the tolerance. This phenomenon might have stemmed from the application of the CCs for the voltage-reactive power relationship. The same situation applies to the change in the active power loss up to the 12th iteration. When the final solution is obtained, the transmission loss is 2.4429 [MW]. Figure 4 shows the change in the active power loss during the solution iteration.

Table 1 shows the reactive power and voltage magnitudes of each generator at the final solution and the corresponding $\mathrm{CC}$ values. The $\mathrm{CC}$ of the generator at bus 5 is related to the maximum reactive power limit, as expressed in 10, and those of the other generators are related to the minimum reactive power limit, as expressed in (11). At the final solution, the CC errors of the generators are different, and the total $\mathrm{CC}$ error is approximately 0.007106 [pu], as shown in the last column in Table 1. This CC error appears to be acceptable from the engineering point of view.

\section{Conclusion}

This paper has presented a fuzzy set method for enforcing $\mathrm{CC}$ in an NIPM-based optimization. NIPM is a Newton-type approach to nonlinear programming problems, but it adopts the log-barrier functions to deal with the obstacle of managing inequality constraints. The fuzzy-enforcement method has been implemented for CCs, which can be incorporated in optimization problems for real-world applications. In this paper,

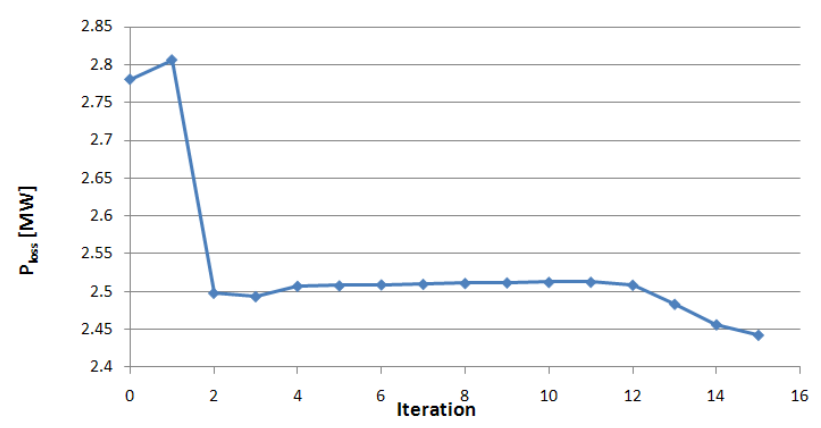

Figure 4. Change in the active power loss during the solution process.

numerical simulations that apply the method to power system $\mathrm{OPF}$ problems have been included.

\section{Conflict of Interest}

No potential conflict of interest relevant to this article was reported.

\section{Acknowledgments}

This work has been supported by Seoul National University of Science \& Technology. 


\section{References}

[1] Z. Q. Luo, J. S. Pang, and D. Ralph, Mathematical Programs with Equilibrium Constraints, Cambridge: Cambridge University Press, 1996.

[2] F. Facchinei and J. S. Pang, Finite-Dimensional Variational Inequalities and Complementarity Problems, New York: Springer-Verlag, 2003.

[3] A. F. Izmailov, "Mathematical programs with complementarity constraints: regularity, optimality conditions, and sensitivity," Computational Mathematics and Mathematical Physics, vol. 44, no. 7. pp. 1145-1164, 2004.

[4] M. C. Ferris and J. S. Pang, "Engineering and economic applications of complementarity problems," SIAM Review, vol. 39, no. 4, pp. 669-713, 1997.

[5] W. Rosehart, C. Roman, and A. Schellenberg, "Optimal power flow with complementarity constraints," IEEE Transactions on Power Systems, vol. 20, no. 2, pp. 813822, May 2005. http://dx.doi.org/10.1109/TPWRS.2005. 846171

[6] S. J. Wright, Primal-Dual Interior Point Methods, Philadelphia: SIAM, 1997.

[7] S. Mehrotra, "On the implementation of a primal-dual interior point method," SIAM Journal on Optimization, vol. 2, no. 4, pp. 575-601, 1992. http://dx.doi.org/10.1137/ 0802028

[8] Y. Nesterov and A. Nemirovskii, Interior-Point Polynomial Algorithms in Convex Programming, Philadelphia: SIAM, 1994.

[9] S. G. Nash and A. Sofer, "On the complexity of a practical interior-point method," SIAM Journal on Optimization, vol. 8, no. 3, pp. 833-849, 1998. http://dx.doi.org/10.1137/ S1052623496306620

[10] A. Forsgren, P. E. Gill, and M. H. Wright, "Interior methods for nonlinear optimization," SIAM Review, vol. 44, no. 4, pp. 525-597, 2002.

[11] K. R. Frisch, The Logarithmic Potential Method Of Convex Programming, Oslo: University Institute of Economics, 1955.
[12] N. Karmarkar, "A new polynomial-time algorithm for linear programming," Combinatorica, vol. 4, no. 4, pp. 373-395, 1984. http://dx.doi.org/10.1007/BF02579150

[13] N. Megiddo, Pathways to the Optimal Set in Linear Programming, San Jose: Technical Report of IBM Almaden Research Center, 1986.

[14] R. D. C. Monteiro and I. Adler, "Interior path following primal-dual algorithms. Part II: convex quadratic programming," Mathematical Programming, vol. 44, no. 1, pp 43-66, May 1989. http://dx.doi.org/10.1007/bf01587076

[15] J. M. Peng and Y. X. Yuan, "Unconstrained method for generalized complementarity problems," Journal of Com putational Mathematics, vol. 15, pp. 253-264, 1997.

[16] H. Wei, H. Sasaki, J. Kubokawa, and R. Yokoyama, "An interior point nonlinear programming for optimal power flow problems with a novel data structure," IEEE Transactions on Power Systems, vol. 13, no. 3, pp. 870-877, Aug. 1998. http://dx.doi.org/10.1109/59.708745

[17] H. Song, B. Lee, S. H. Kwon, and V. Ajjarapu, "Reactive reserve-based contingency constrained optimal power flow (RCCOPF) for enhancement of voltage stability margins,' IEEE T Transactions on Power Systems, vol. 18, no. 4, pp. 1538-1546, Nov. 2003. http://dx.doi.org/10.1109/TPWRS. 2003.818759

[18] W. H. E. Liu and X. Guan, "Fuzzy constraint enforcement and control action curtailment in an optimal power flow,' IEEE Transactions on Power Systems, vol. 11, no. 2, pp. 639-644, May 1996. http://dx.doi.org/10.1109/59.496133

[19] H. J. Zimmerman, Fuzzy Set Theory and Its Application, 2nd ed., Boston: Kluwer Academic Publishers, 1991.

[20] H. Song, R. Dosano, and B. Lee, "Power system voltage stability classification using interior point method based support vector machine (IPMSVM)," International Journal of Fuzzy Logic and Intelligent Systems, vol. 9, no. 3, pp. 238-243, Sep. 2009.

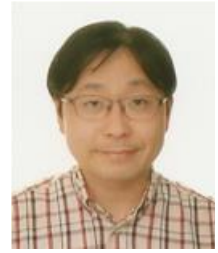

Hwachang Song received B.S., M.S., and Ph.D. degrees in Electrical Engineering from Korea University in 1997, 1999, and 2003 , respectively. He was Post-doctoral Visiting Scholar at Iowa State University, U.S., from 2003 to 2004. He was Faculty Member with the School of 
Electronic and Information Engineering, Kunsan National University, Korea, from 2005 to 2008. Currently, he is Associate Professor with the Department of Electrical and Information Engineering, Seoul National University of Science \& Technology, Korea. His research interests include nonlinear optimization, power system stability and control, battery energy storage systems, and system modeling.

Tel: +82-2-970-6402

E-mail: hcsong@seoultech.ac.kr 